\title{
Functional protease profiling for laboratory based diagnosis of invasive aspergillosis
}

\author{
BASSEL SABBAGH ${ }^{1}$, VICTOR COSTINA ${ }^{1}$, DIETER BUCHHEIDT ${ }^{2}$, MARK REINWALD $^{2}$, \\ MICHAEL NEUMAIER $^{1}$ and PETER FINDEISEN ${ }^{1}$ \\ ${ }^{1}$ Institute for Clinical Chemistry, ${ }^{2}$ Department of Haematology and Oncology, Medical Faculty Mannheim \\ of the University of Heidelberg, University Hospital Mannheim, D-68167 Mannheim, Germany
}

Received February 19, 2015; Accepted March 27, 2015

DOI: $10.3892 /$ ijo.2015.2984

\begin{abstract}
Invasive aspergillosis (IA) remains difficult to diagnose in immunocompromised patients, because diagnostic criteria according to EORTC/MSG guidelines are often not met and have low sensitivity. Hence there is an urgent need to improve diagnostic procedures by developing novel approaches. In the present study, we present a proof of concept experiment for the monitoring of Aspergillus associated protease activity in serum specimens for diagnostic purpose. Synthetic peptides that are selectively cleaved by proteases secreted from Aspergillus species were selected from our own experiments and published data. These so called reporter peptides $(\mathrm{RP}, \mathrm{n}=5)$ were added to serum specimens from healthy controls $(\mathrm{HC}, \mathrm{n}=101)$ and patients with proven (IA, $\mathrm{n}=9$ ) and possible (PIA, n=144) invasive aspergillosis. Spiked samples were incubated ex vivo under strictly standardized conditions. Proteolytic fragments were analyzed using MALDI-TOF mass spectrometry. Spiked specimens of IA patients had highest concentrations of RP-fragments followed by PIA and HC. The median signal intensity was 116.546 (SD, 53.063) for IA and 5.009 (SD, 8.432) for HC. A cut-off $>36.910$ was chosen that performed with $100 \%$ specificity and sensitivity. Patients with PIA had either values above [53\% (76/144)]
\end{abstract}

Correspondence to: Professor Peter Findeisen, Institute for Clinical Chemistry, Medical Faculty Mannheim of the University of Heidelberg, University Hospital Mannheim, Theodor Kutzer Ufer 1-3, D-68167 Mannheim, Germany

E-mail: peter.findeisen@umm.de

Abbreviations: IA, invasive aspergillosis; PIA, possible invasive aspergillosis; HC, healthy controls; MALDI-TOF MS, matrix assisted laser desorption ionisation time of flight mass spectrometry; $\mathrm{RP}$, reporter peptide; RPF, reporter peptide fragment; CV, coefficient of variation; SD, standard deviation; ACCS, Aspergillus fumigatus cell culture supernatant; EORTC/MSG, European Organization for Research and Treatment of Cancer/Invasive Fungal Infections Cooperative Group and the National Institute of Allergy and Infectious Diseases Mycoses Study Group

Key words: haemato-oncology, neutropenia, invasive aspergillosis, MALDI-TOF mass spectrometry, serum, proteases or below [47\% (67/144)] this chosen cut-off. The detection of respective reporter peptide fragments can easily be performed by MALDI TOF mass spectrometry. In this proof of concept study we were able to demonstrate that serum specimens of patients with IA have increased proteolytic activity towards selected reporter peptides. However, the diagnostic value of functional protease profiling has to be validated in further prospective studies. It is likely that a combination of existing and new methods will be required to achieve optimal performance for diagnosis of IA in the future.

\section{Introduction}

The ubiquitous fungus Aspergillus fumigatus is the predominant cause of life-threatening invasive aspergillosis (IA) in immunocompromised patients but further Aspergillus species such as A. niger and A. flavus can also cause invasive disease (1). Over the last decades, there has been a substantial increase in the number of immunocompromised persons concomitant with an increase in the number of IA related deaths (2). While exposure of immunocompetent individuals to conidia remains with no further implications, it may become lethal in immunocompromised patients (3). Hence, IA is the leading cause of death in patients with acute leukemia and recipients of allogeneic haematopoietic stem-cell transplants (2). Furthermore, patients with advanced HIV infections and patients undergoing immuno-modulation therapies or chemotherapy are also at risk to acquire IA (4).

Symptoms which have been linked to IA are non-specific; this comprises cough, chest pain and fever and patients might even remain asymptomatic (5). Accordingly, early and accurate diagnosis of IA is mandatory in order to improve the patient outcome and restrain utilization of antifungal drugs prescribed for patients at high risk of IA. Unfortunately, currently applied diagnostic methods of Aspergillus infections lack sensitivity and/or specificity.

Diagnostic tools based on ELISA such as the detection of the cell wall components galactomannan (GM) or (1->3)$\beta$-D-glucan (BDG) are widely used in the diagnosis of IA (6). Nevertheless, many factors can compromise the results of both assays. The accuracy of GM testing can be altered by antibiotic (7) or antifungal therapy (8) and inadequate sampling strategies (6). In addition, both GM and BDG are not characteristic 
for fungi of the genus Aspergillus and are also produced from other species in the fungal kingdom $(9,10)$. False positive BDG results have also been reported in patients treated with immunoglobulins or antibiotics such as amoxicillin (11). PCR based diagnosis is a promising method for detecting Aspergillus DNA, due to high sensitivity and specificity. Nevertheless, there are some constrains for routine laboratory use including lack of standardized protocols, reproducibility and risk of contamination (12).

We hypothesized, that the monitoring of Aspergillus specific proteolytic acitivity in serum specimens might be an alternative approach for diagnosis of IA. In immunocompromised patients the airborne conidia can germinate and produce a branched mycelium that penetrates the lung tissue invading blood vessels and spreading hematogenously. During invasion several toxic molecules as well as enzymes including a variety of proteases are secreted. These proteases play a crucial role in the breakdown of the extracellular matrix barrier of the host (13). Recently, Aspergillus associated protease activity has been characterized in bronchoalveolar lavage (BAL) using fluorogenic substrates (14). Due to the high pulmonary blood flow it is most likely that secreted proteases are also present in serum specimens that can be obtained much easier than BAL fluid. In a previous study we have already demonstrated the feasibility of protease profiling for the detection of disease associated proteolytic activity in serum specimens. Synthetic peptides designated as reporter peptides (RP) are added to serum specimens and the accumulation of cleaved products is indicating disease associated proteolytic activity. The main advantages of RP spiking is the ease of standardization making this procedure highly reproducible (15). Furthermore, the enzymatic cleavage of RPs in combination with mass spectrometry based quantitation of RP-fragments leads to high diagnostic sensitivity $(16,17)$. Here, we selected five different reporter peptides that are exclusively cleaved by proteases secreted from Aspergillus species. These RPs were added to serum specimens of patients with invasive aspergillosis (IA), possible invasive aspergillosis (PIA) and healthy controls (HC) and incubated under standardized conditions prior to MALDI-TOF MS analysis. As expected, the specimens from patients with IA showed highest concentrations of RP-fragments indicating increased activity of Aspergillus specific proteases. This functional protease profiling might be a promising approach for laboratory based diagnosis of IA. However, our preliminary results of this proof of concept experiment have to be validated thoroughly in future studies.

\section{Materials and methods}

Aspergillus culture. Clinical isolates of A.fumigatus were grown on Sabouraud dextrose agar (Oxoid Ltd., Hampshire, UK) at $30^{\circ} \mathrm{C}$ for at least 7 days. After spore formation had occurred, conidia were harvested manually using a pipette tip and inoculated in $50 \mathrm{ml}$ of Aspergillus minimal medium (AMM) containing $500 \mu \mathrm{l}$ of a $100 \mathrm{X}$ penicillin/streptomycin solution (final concentration: $0.1 \mathrm{mg} / \mathrm{ml}$ each) and liquid cultures were incubated at $37^{\circ} \mathrm{C}$. Supernatant of cultures was harvested after 4 days, filtered through a syringe filter (Fisher Scientific GmbH, Schwerte, Germany; size cut-off $0.22 \mu \mathrm{m}$ ) and stored directly at $-80^{\circ} \mathrm{C}$.
Table I. Patient characteristics.

\begin{tabular}{|c|c|c|c|}
\hline EORTC/MSG & Gender & Age (years) & Diagnosis \\
\hline \multirow[t]{5}{*}{ IA } & Male & 47 & NHL \\
\hline & Female & 59 & AML \\
\hline & Male & 50 & AML \\
\hline & Male & 27 & CML \\
\hline & Female & 25 & CML \\
\hline \multirow[t]{16}{*}{ PIA } & Male & 74 & AML \\
\hline & Male & 55 & AML \\
\hline & Female & 62 & CML \\
\hline & Male & 45 & AML \\
\hline & Female & 68 & AML \\
\hline & Male & 50 & AML \\
\hline & Female & 58 & AML \\
\hline & Female & 51 & AML \\
\hline & Female & 67 & AML \\
\hline & Female & 69 & AML \\
\hline & Female & 44 & AML \\
\hline & Female & 40 & AML \\
\hline & Female & 25 & AML \\
\hline & Female & 44 & AML \\
\hline & Male & 56 & AML \\
\hline & Female & 51 & AML \\
\hline
\end{tabular}

EORTC/MSG criteria: IA, invasive aspergillosis; PIA, possible invasive aspergillosis; EORTC/MSG, European Organization for Research and Treatment of Cancer/Invasive Fungal Infections Cooperative Group and the National Institute of Allergy and Infectious Diseases Mycoses Study Group; CML, chronic myeloid leukemia; AML, acute myeloid leukemia; NHL, non-Hodgkin's lymphoma.

Patients and blood collection. Patients were classified based on the EORTC/MSG consensus criteria (18). Nine serum samples from five patients with proven invasive aspergillosis (IA) were obtained from the Department of Internal Medicine $\mathrm{C}$ of the University of Greifswald, Germany. Furthermore, ten serum specimens of these five patients were obtained prior to the diagnosis of IA when patients were classified as having a possible invasive aspergillosis. The number of blood specimens per patient ranged from 1 to 6 and longitudinal blood sampling was consecutively performed within a time range of up to 240 days prior to diagnosis of IA. Blood from 101 healthy control individuals (HC) was taken from voluntary employees of the University Hospital Mannheim during routine laboratory testing at the occupational medicine. Furthermore, 144 blood samples of 16 immunosuppressed patients with haematological malignancies that were classified as having a possible invasive aspergillosis (PIA) were also integrated into the study. The number of blood specimens per patient ranged from 1 to 22 and longitudinal blood sampling was consecutively performed within a time range of up to 185 days. The characteristics of patients are summarized in Table I. 
Table II. Reporter peptide sequences.

\begin{tabular}{lrr}
\hline Name & Aa sequence & {$[\mathrm{M}+\mathrm{H}]^{+}$} \\
\hline RP1 & Bio-Abu-Ahx-RPKPVE-Nva-WREAKdHHHHHH & $2,858.4$ \\
RPF1 & KdHHHHHH & $1,084.5$ \\
RP2 & Bio-Abu-Ahx-WKPYDAADLtnHHHHHHt & $2,640.8$ \\
RPF2 & DAADLtnHHHHHHt & $1,642.7$ \\
RP3 & Bio-Abu-Ahx-CGSHLVEAttHHHHHH & $2,264.8$ \\
RPF3 & AttHHHHHH & $1,114.5$ \\
RP4 & Bio-Abu-Ahx-DGVDLKTQyHHHHHHn & $2,399.9$ \\
RPF4 & DLKTQyHHHHHHn & $1,703.8$ \\
RP5 & Bio-Abu-Ahx-GFFYTPKAvHHHHHHn & $2,391.3$ \\
RPF5 & AvHHHHHHn & $1,125.5$ \\
\hline
\end{tabular}

RP, reporter peptide; RPF, reporter peptide fragment; Aa, amino acid; Abu, aminobutyric acid; Ahx, aminohexanoic acid. Lower case characters, d-amino acids.

Blood collection was performed after we obtained institutional review board approval from the local ethics committee and patients' written informed consent. Whole blood $(10 \mathrm{ml})$ was collected in serum tubes (Sarstedt AG \& Co., NümbrechtRommelsdorf, Germany). Samples were kept for $30 \mathrm{~min}$ at room temperature prior to centrifugation at $3,000 \mathrm{x} \mathrm{g}$ (10 min, $20^{\circ} \mathrm{C}$ ). Finally, the serum was removed, divided in aliquots and stored at $-80^{\circ} \mathrm{C}$ until further use.

Galactomannan assay. Serum galactomannan (GM) levels were measured using the Platelia Aspergillus enzyme immunoassay test (Bio-Rad Laboratories GmbH, Munich, Germany) according to the manufacturer's instructions. Results were recorded as the ratio of optical density of the sample to that of threshold control samples. Cut-off value of GM was 0.5 of optical density index (ODI) as approved by FDA (19).

Reporter peptide synthesis and spiking. Peptide sequences were selected from our recent study $(20,21)$ as well as publicly available data $(22,23)$ and were synthesized by the Peptide Synthesis Facility of the German Cancer Research Center (Heidelberg, Germany). The reporter peptides (RP) and respective reporter peptide fragments (RPF) are shown in Table II. Any RP was prepared as a $4 \mathrm{mmol} / \mathrm{l}$ stock solution in DMSO. RPs were diluted to $100 \mu \mathrm{mol} / 1$ in PBS pH 7.4 (PAA Laboratories). Each serum sample was diluted 1:3 in PBS. For the proteolytic profiling, $50 \mu \mathrm{l}$ of diluted serum sample was mixed with $50 \mu \mathrm{l} \mathrm{RP}$ solution and incubated at $37^{\circ} \mathrm{C}$ for $24 \mathrm{~h}$.

Purification of proteolytic fragments. As shown in a previous study, the analytical sensitivity of protease profiling can substantially be enhanced using serial affinity chromatography of doubly tagged RPs (16). Accordingly, the full length RP was depleted prior to enrichment of His-tagged C-terminal part of cleaved RPs and subsequent MALDI-TOF MS (Fig. 1). Streptavidin sepharose high-performance slurry (GE Healthcare, Uppsala, Sweden) was washed twice and resuspended in PBS pH 7.4 to yield a 50\% slurry. We then added $50 \mu \mathrm{l}$ streptavidin slurry to each spiked sample and incubated the mixture for $15 \mathrm{~min}$ at room temperature.
After binding, the streptavidin sepharose was centrifuged at $1,100 \times \mathrm{g}$ for $5 \mathrm{~min}$ and discarded. A $20-\mu 1$ aliquot of the supernatant was mixed with $60 \mu \mathrm{l}$ binding buffer and $10 \mu \mathrm{l}$ IMAC SL (immobilized metal ion affinity chromatography on self-loading) magnetic beads (Bruker Daltonik GmbH, Bremen, Germany) that were loaded with $\mathrm{Ni}^{2+}$ ions, and then further processed according to the manufacturer's instructions. Briefly, the samples were incubated for $30 \mathrm{~min}$ at room temperature, washed 2 times each with 3 different buffers (100 $\mu 1$ per wash step), and eluted with $10 \mu l$ elution buffer for $15 \mathrm{~min}$ at room temperature.

MALDITOF-mass spectrometry. We diluted $1 \mu 1$ of each eluted sample with matrix solution (1:10) and $1 \mu \mathrm{l}$ of the resulting mixture was spotted onto a SCOUT 600- $\mu$ m AnchorChip target (Bruker Daltonik $\mathrm{GmbH}$ ). The matrix solution was prepared by dissolving $0.3 \mathrm{~g} / 1$ of $\alpha$-cyano-4-hydroxycinnamic acid solution (Bruker Daltonik $\mathrm{GmbH}$ ) in ethanol/acetone (2:1, $\mathrm{vol} / \mathrm{vol}$ ), and external mass calibration was then performed with a peptide calibration standard (Bruker Daltonik $\mathrm{GmbH}$ ). MALDI-TOF MS measurements were performed using an Autoflex II (Bruker Daltonik GmbH) operating in the positive mode. The MS spectra for peaks in the range of 1-3 kDa were generated by summarizing 500 laser shots ( 50 laser shots at 10 different spot positions). Peak detection was performed using the centroid algorithm. The peak intensities were normalized to the total ion count of the respective mass spectra. For data acquisition and analysis, the software packages FlexControl ${ }^{\mathrm{TM}}$ 2.2 and Flex Analysis ${ }^{\mathrm{TM}} 2.2$ (both from Bruker Daltonik $\mathrm{GmbH})$ were used.

Reproducibility. The 1:3 mixtures of Aspergillus fumigatus cell culture supernatant and healthy control serum was aliquoted to generate the specimens designated as 'quality control' (QC). All aliquots were kept at $-80^{\circ} \mathrm{C}$ until further use. Quality control samples were further on handled similarly to the patient specimens and regularly interspersed into analytical runs to determine reproducibility of reporter peptide spiking. All samples were strictly randomized prior to further handling, processing and analysis. 


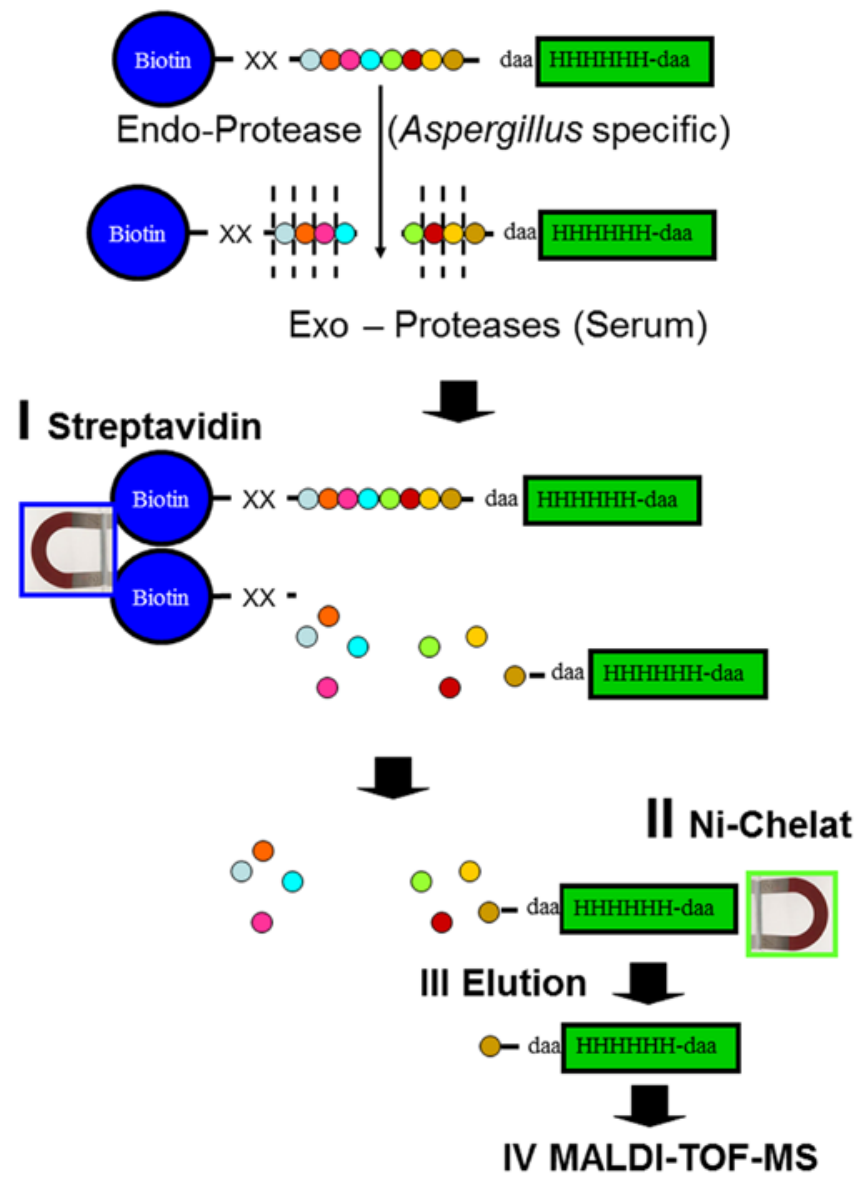

Figure 1. Schematic workflow of peptide extraction with serial affinity chromatography. Reporter peptides are added to serum specimens. After incubation for $24 \mathrm{~h}$ at $37^{\circ} \mathrm{C}$ the unprocessed reporter peptides and proteolytic fragments that are labelled with biotin are depleted with streptavidin sepharose (I). The remaining 6His-tag labelled anchorpeptides are extracted with Ni-chelate beads (II). After elution (III) the peptides are analyzed with MALDI-TOF MS (IV). XX, linker; daa; d-aminoacid.

Statistical analysis. Coefficients of variation (CV) and standard deviations (SD) were calculated using Microsoft Excel 2010 (Microsoft software). The data visualization using whisker-box plots was performed with MedCalc ${ }^{\mathrm{TM}}$ software, version 13.1.1 (MedCalc Software, Ostend, Belgium).

\section{Results}

Specificity of RP spiking. Reporter peptides were added to specimens and incubated prior to MALDI-TOF MS as described in Materials and methods. The cleavage pattern of Aspergillus fumigatus cell culture supernatant (ACCS), serum of healthy controls (HC) and the mixture of ACCS and $\mathrm{HC}$ were compared to each other. Any of the five RPs was cleaved in ACCS and the mixture of ACCS and HC and respective proteolytic fragments (Table II) were prominent in subsequent MALDI-TOF MS analyses. In contrast, after incubation of RPs in HC serum the respective proteolytic fragments were almost undetectable. This indicates that RPs are selectively cleaved by secreted proteases of Aspergillus fumigatus whereas they are stable in serum specimens of healthy controls. The representative MS-profiles of RP5 are exemplarily shown in Fig. 2.
Reproducibility. The reproducibility of RP spiking was tested with ten quality control specimens (QC) that were randomly interspersed into measurements of serum from patients and controls. As expected, the coefficient of variation (CV) was inversely correlated to the signal intensity of reporter peptide fragments (RPFs) (24). Lowest CV of $8 \%$ was found for RPF5 with median signal intensity of 55.040 a.U. (SD 4.132). Highest $\mathrm{CV}$ of $31 \%$ was found for RPF2 with median signal intensity of 20.150 a.U. (SD 6.292).

Reporter peptide processing in serum specimens from patients with invasive aspergillosis and controls. Any of the five RPs was incubated in serum specimens of healthy controls (HC, $n=101)$ and patients with proven invasive aspergillosis (IA, n=9) as described in Materials and methods. The signal intensities of reporter peptide fragments 1-5 were significantly higher in IA serum specimens when compared to HC (Fig. 3).

For reason of simplification the signal intensities of RPFs were summarized and plotted over time to demonstrate that longitudinal sampling is essential for reporter peptide spiking. As shown in Fig. 4 there is a strong accumulation of RPFs next to the time-point ( -6 to +4 days) of diagnosis of proven IA (time-point zero in Fig. 4). In contrast, specimens that were obtained in a time period ranging from 43 to 240 days prior to diagnosis of IA showed low signal intensities for RPFs. Furthermore, these specimens showed no statistically relevant difference in RPF signal intensities when compared to healthy controls (data not shown).

Cut-off calculation. The median signal intensity of IA specimens was 116.546 (SD, 53.063) with maximal and minimal values of 190.943 and 39.197, respectively. The median signal intensity of HC specimens was 5.009 (SD, 8.432) with maximal and minimal values of 36.910 and 210, respectively. As there is no overlap of values between the two collectives a cut-off $>36.910$ was chosen that performed with $100 \%$ diagnostic specificity and sensitivity.

Reporter peptide processing in serum specimens from patients with possible IA. Reporter peptides were added to serum specimens $(\mathrm{n}=144)$ of 16 patients that were classified as having a possible invasive aspergillosis (PIA) at the time-point of blood withdrawal. Samples were processed as described in Materials and methods prior to MALDI-TOF mass spectrometry. From a total of 144 PIA samples $18 \%(26 / 144)$ were positive and $65 \%(93 / 144)$ were negative for galactomannan (GM). For $17 \%$ $(25 / 144)$ of serum specimens no GM values were available. PIA samples with positive GM values had significantly higher mass spectrometric signal intensities of reporter peptide fragments (median, 56.235; SD, 26.298) when compared to those that were negative for GM (median, 28.781; SD, 30.678). As already mentioned the signal intensities of reporter peptide fragments showed highest values in IA specimens and lowest values in $\mathrm{HC}$ specimens with no overlap. In contrast, signal intensity of PIA specimens was between these two collectives and has overlap with IA and $\mathrm{HC}$ collectives, respectively (Fig. 5). With reporter peptide spiking 53\% of specimens (76/144) could be classified as positive and $47 \%(68 / 144)$ as negative when the cut-off of 36.910 a.U. for sum of RPF signals was applied. However, the longitudinal monitoring of these 16 


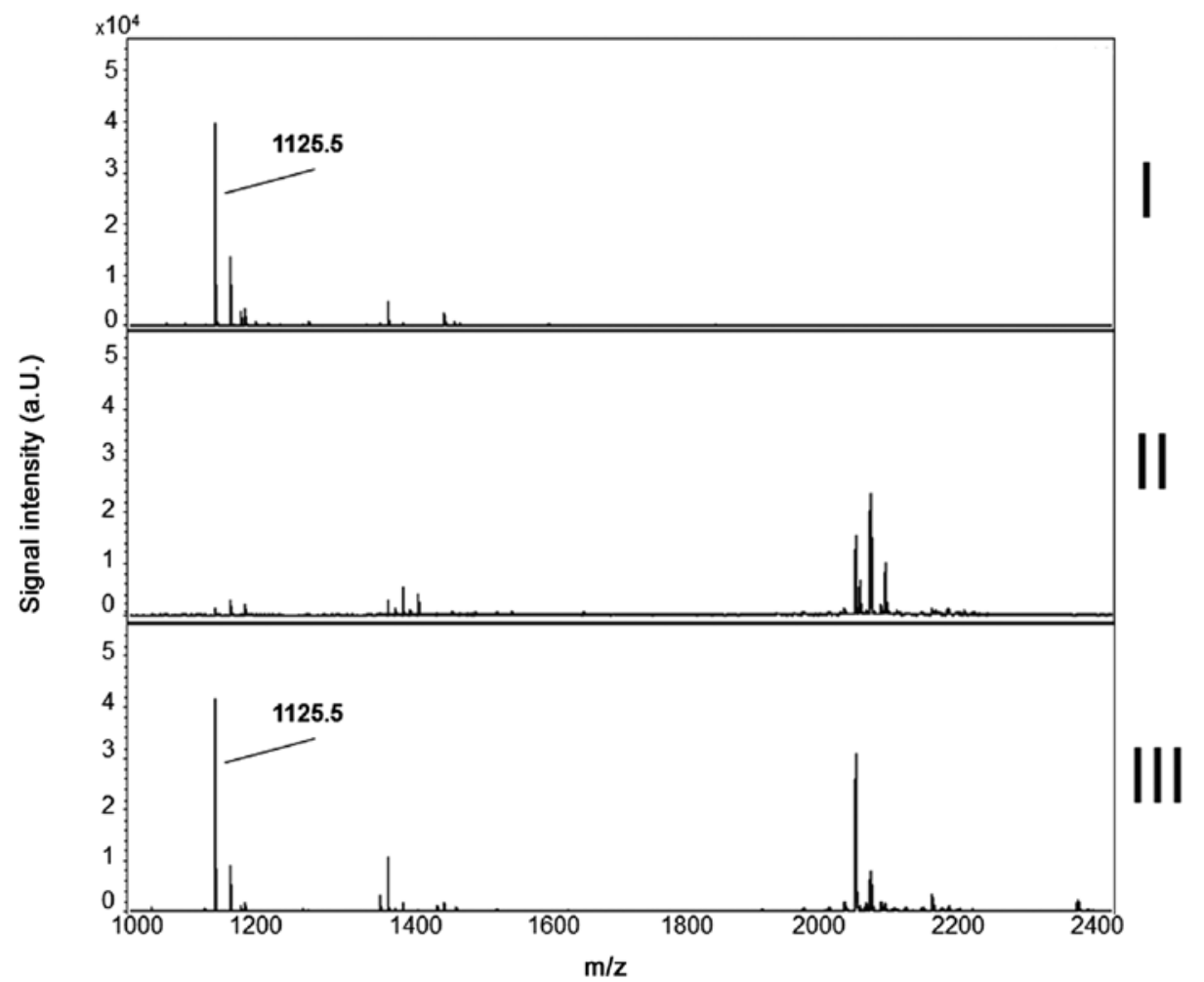

Figure 2. Exemplary MS profiles after incubation of RP5 in (I) Aspergillus fumigatus cell culture supernatant (ACCS), (II) serum specimen of healthy control (HC) and (III) mixture of ACCS and HC (1:3). RP-fragments were extracted by serial affinity chromatography prior to MALDI-TOF MS as described in Materials and methods.

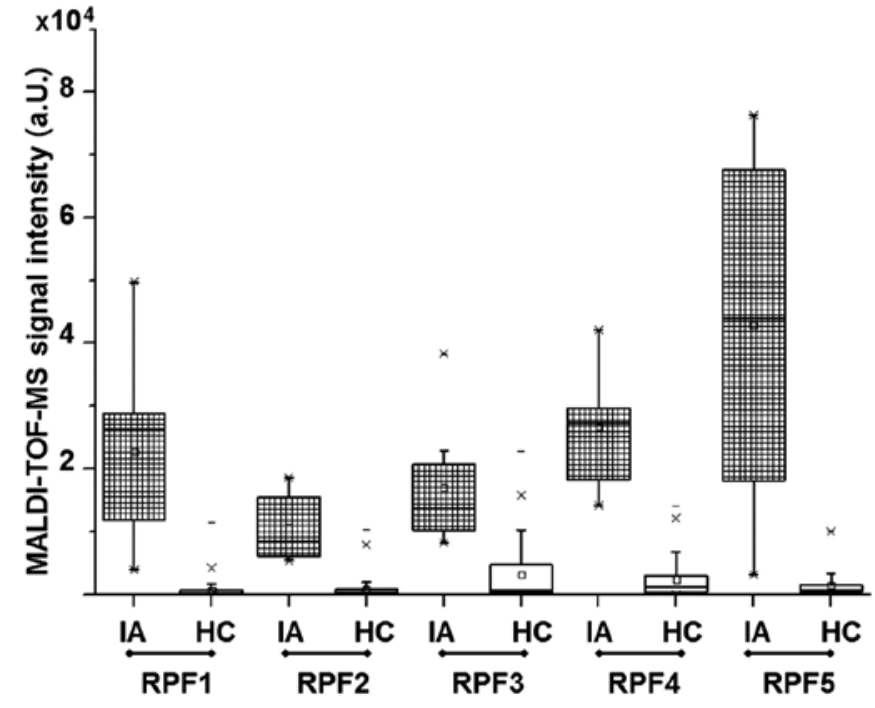

Figure 3. Reporter peptide fragment (RPF) signal intensity (a.U.) in serum of healthy controls (HC) and patients with proven invasive aspergillosis (IA). The composition of RPF1-5 is displayed in Table II. RPs (1-5) were incubated with serum specimens and RPFs were serially extracted prior to MALDI-TOF MS as described in Materials and methods.

patients with RP spiking revealed a heterogeneous pattern of proteolytic activity (Fig. 6). One patient had completely negative values over time (patient 6, Fig. 6A) for the chosen cut-off. In contrast few other patients had completely positive values over time (e.g. patient 3, Fig. 6A). Most patients showed undulating graphs with positive and negative values and clear

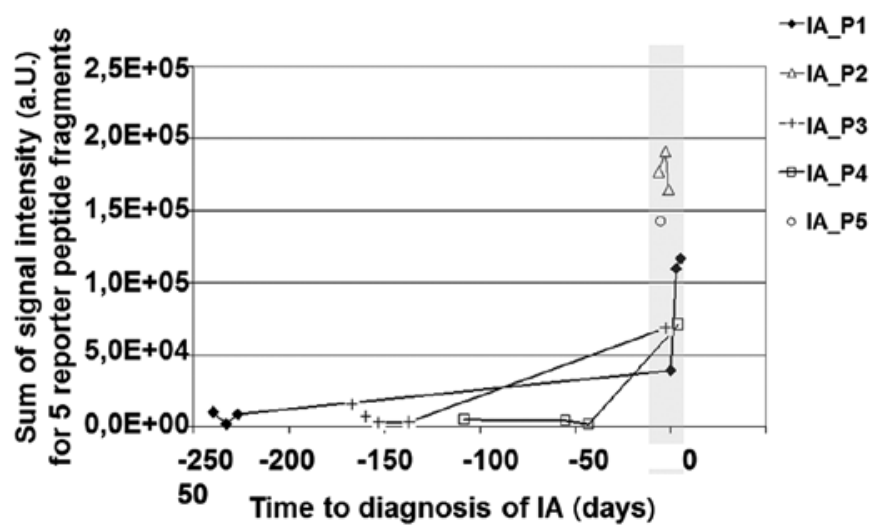

Figuer 4. Longitudinal monitoring of reporter peptide fragment (RPF) signal intensity for 5 patients with proven invasive aspergillosis. Time-point zero; time of proven IA diagnosis. Grey underlined; timeframe of -6 to +4 days in relation to diagnosis of proven IA (time-point zero).

maxima with signal intensities $>100.000$ a.U. were observed (e.g. patient 13, Fig. 6B). However, for in-depth interpretation of longitudinal changes additional data, specifically prompt chest CT scan or Aspergillus antigen testing from BAL would be necessary, but were not available for this cohort of patients.

\section{Discussion}

Since diagnostic tools lack sensitivity or specificity, the final diagnosis of IA is rather frequently confirmed only during autopsy (25). Accordingly, there is pressing need for the development of rapid and accurate diagnostic tests. In the 


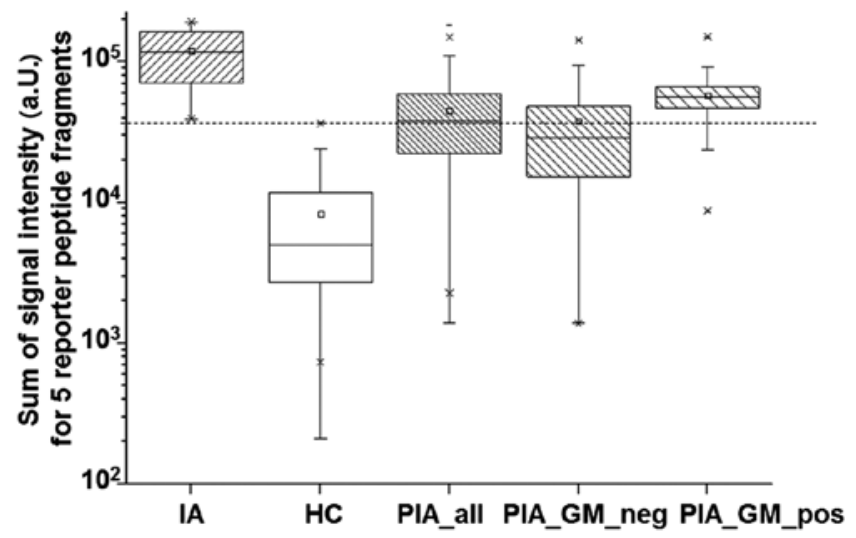

Figure 5. Reporter peptide fragment (RPF) signal intensity in the cohort of patients with proven invasive aspergillosis (IA), healthy controls (HC), patients with possible invasive aspergillosis (PIA_all, $n=144$ ) that in addition were subdivided into those with negative (PIA_GM_neg, n=93/144) and positive (PIA_GM_pos, n=26/144) galactomannan assay. For 24 of 144 PIA cases no galactomannan (GM) results were available.

present study we applied a mass spectrometry based method using reporter peptide spiking for monitoring of Aspergillus specific proteolytic activity in serum specimens of healthy controls and patients with proven or possible IA according to the EORTC/MSG criteria.
Multiple proteases are secreted from Aspergillus species during the course of infection (13). We previously demonstrated that disease specific proteolytic activity can be detected in serum specimens of respective patients (reviewed in ref. 26). Accordingly, proteases have been proposed as novel biomarkers for diagnosis of IA $(14,20,22)$. Spiking of clinical specimens with synthetic reporter peptides offers an efficient diagnostic tool for monitoring distinct proteolytic activity in clinical specimens. For example, the activity of multiple coagulation factors can be quantitatively assessed by the use of chromogenic substrates (27). These are cleaved by the protease of interest e.g. the serine protease coagulation factor $\mathrm{X}$ that has high affinity towards a synthetic substrate (S-2222). The cleavage of S-2222 is inversely correlated to the heparin concentration that inhibits factor X activity (27).

Mass spectrometry (MS)-based proteomic profiling can easily be applied to a variety of clinical specimens such as serum, plasma or tissue samples. MALDI-TOF MS has been typically performed due to its high sample throughput capacity, robustness and ease of use. However, the combination of different RPs for multiplex analysis has limitations due to ion suppression which could hamper the detection of peptides and lead to an incomplete recovery of proteolytic fragments (26). In order to minimize negative effects we applied a tandem affinity purification protocol for extraction of RPFs as recently described (16). Furthermore, the synthetic reporter peptides
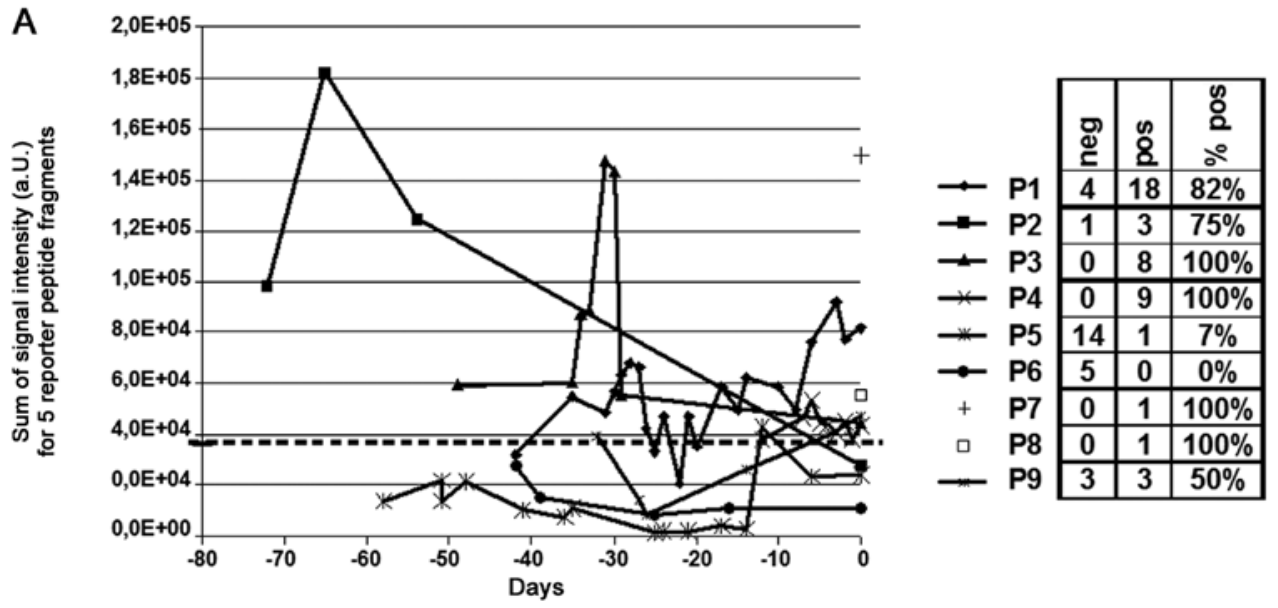

B
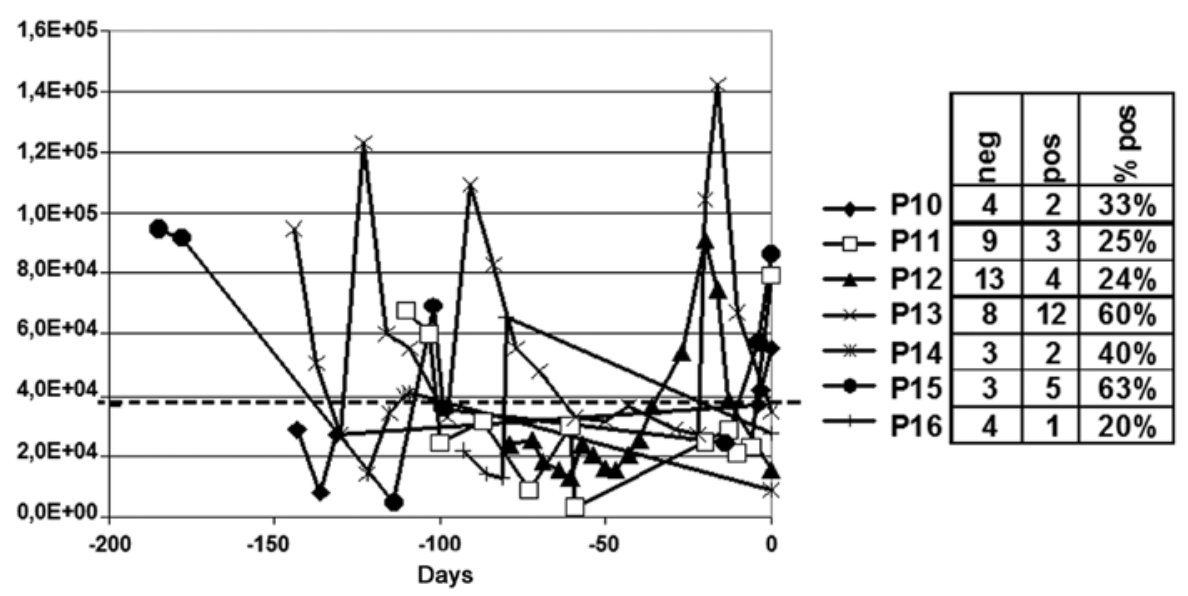

Figure 6. Longitudinal monitoring of RPF signal intensity for patients with possible invasive aspergillosis. (A) Patients (P) 1-9. (B) Patients (P) 10-16. Time-point zero represents the most recent data of blood withdrawal. 
were stabilized by introducing $\mathrm{D}$-amino acids to avoid unwanted processing by exoproteases that contribute to the intrinsic proteolytic activity of serum specimens $(28,29)$.

In the present study, we validated a set of five RPs for laboratory-based diagnosis of IA. We used a relatively small number of serum specimens for this proof-of-concept experiment, because obtaining serum specimens from patients with proven IA is difficult. We were able to obtain only 9 serum specimens from 5 patients with proven IA. The numerical imbalance of IA specimens $(n=9)$ compared to $\mathrm{HC}(\mathrm{n}=101)$ and PIA $(n=144)$ samples is an obvious limitation of the present study.

The EORTC/MSG criteria were defined to increase standardization and allowed results of studies of invasive fungal disease to be interpreted and compared more uniformly (30). However, one of the major hurdles when performing these criteria is that most patients are classified as possible cases, whereas probable or proven IA cases are rare (31-33). For example, Subirà et al (32) performed a diagnostic study on 22 patients in order to test the applicability of the EORTC/MSG criteria. All of the patients in the present study had haematological malignancies and IA at autopsy. However, results showed large differences between ante- and post-mortem classifications. Only 2 patients had been classified as proven while they were alive, 6 as probable, 13 as possible and 1 remained unclassified. In addition, $64 \%$ of these patients had no microbiological or major clinical criteria. Hence, even the 2008 EORTC/MSG criteria still have a low sensitivity rendering the correct classification of patients an unsolved and serious problem for clinical studies $(32,33)$.

Accordingly, it may be presumed, that our collective of possible IA $(n=144)$ also comprises cases of probable or proven IA as well. Our results for functional protease profiling are indicating that $53 \%(76 / 144)$ have values above the chosen cut-off that separates proven IA cases from healthy controls. Furthermore, higher concentrations of RPFs as surrogate marker for Aspergillus specific protease activity are associated with positive galactomannan assays as another surrogate marker for IA (Fig. 5). In this retrospective study the patients' EORTC/MSG status cannot be elucidated definitely and reclassification attempts are highly speculative. However, functional protease profiling can be performed from a simple blood specimen and longitudinal assaying in a future prospective study seems to be feasible. Laboratory results should be combined with other diagnostic approaches such as imaging (chest CT scan) or Aspergillus antigen testing from BAL to enable a more profound interpretation of the data (34). Taken together, MS-based functional protease profiling is promising for IA diagnosis and could foster the diagnostic yield of IA when it is combined with other existing approaches, and diagnostic accuracy should be evaluated in further prospective studies.

\section{Acknowledgements}

The present study was supported by a grant from the Deutsche José Carreras Leukämiestiftung (Proposal no. DJCLS R 11/11). We thank Dr William Krüger from the Department of Internal Medicine C of the Ernst-Moritz-Arndt-University Greifswald, Germany for providing specimens from patients with proven IA. Furthermore, we thank Dr Klaus Becker from the Institute for Microbiology of the Medical Faculty Mannheim of the University of Heidelberg, for providing cell culture supernatants of Aspergillus species and results of Galactomannan assays.

\section{References}

1. Denning DW: Invasive aspergillosis. Clin Infect Dis 26: 781-803, quiz 804-805, 1998

2. Segal BH: Aspergillosis. N Engl J Med 360: 1870-1884, 2009.

3. Hohl TM and Feldmesser M: Aspergillus fumigatus: Principles of pathogenesis and host defense. Eukaryot Cell 6: 1953-1963, 2007.

4. Thompson GR III and Patterson TF: Pulmonary aspergillosis: Recent advances. Semin Respir Crit Care Med 32: 673-681, 2011.

5. Latgé JP: Aspergillus fumigatus and aspergillosis. Clin Microbiol Rev 12: 310-350, 1999.

6. Hope WW, Walsh TJ and Denning DW: Laboratory diagnosis of invasive aspergillosis. Lancet Infect Dis 5: 609-622, 2005.

7. Adam O, Aupérin A, Wilquin F, Bourhis JH, Gachot B and Chachaty E: Treatment with piperacillin-tazobactam and falsepositive Aspergillus galactomannan antigen test results for patients with hematological malignancies. Clin Infect Dis 38: 917-920, 2004.

8. McCulloch E, Ramage G, Rajendran R, Lappin DF, Jones B, Warn P, Shrief R, Kirkpatrick WR, Patterson TF and Williams C: Antifungal treatment affects the laboratory diagnosis of invasive aspergillosis. J Clin Pathol 65: 83-86, 2012.

9. Barton RC: Laboratory diagnosis of invasive aspergillosis: From diagnosis to prediction of outcome. Scientifica Cairo 2013: 459405, 2013.

10. Pickering JW, Sant HW, Bowles CA, Roberts WL and Woods GL: Evaluation of a (1->3)-beta-D-glucan assay for diagnosis of invasive fungal infections. J Clin Microbiol 43: 5957-5962, 2005.

11. Mennink-Kersten MASH, Warris A and Verweij PE: 1,3- $\beta$-Dglucan in patients receiving intravenous amoxicillin-clavulanic acid. N Engl J Med 354: 2834-2835, 2006.

12. Einsele $\mathrm{H}$ and Loeffler $\mathrm{J}$ : Contribution of new diagnostic approaches to antifungal treatment plans in high-risk haematology patients. Clin Microbiol Infect 14 (Suppl 4): 37-45, 2008.

13. Kogan TV, Jadoun J, Mittelman L, Hirschberg K and Osherov N: Involvement of secreted Aspergillus fumigatus proteases in disruption of the actin fiber cytoskeleton and loss of focal adhesion sites in infected A549 lung pneumocytes. J Infect Dis 189: 1965-1973, 2004.

14. Jambunathan K, Watson DS, Najvar LK, Wiederhold NP, Kirkpatrick WR, Patterson TF, Askew DS, Kodukula K and Galande AK: Prolyl endopeptidase activity in bronchoalveolar lavage fluid: A novel diagnostic biomarker in a guinea pig model of invasive pulmonary aspergillosis. Med Mycol 51: 592-602, 2013.

15. Findeisen P, Post S, Wenz F and Neumaier M: Addition of exogenous reporter peptides to serum samples before mass spectrometry-based protease profiling provides advantages over profiling of endogenous peptides. Clin Chem 53: 1864-1866, 2007.

16. Peccerella T, Lukan N, Hofheinz R, Schadendorf D, Kostrezewa M, Neumaier M and Findeisen P: Endoprotease profiling with double-tagged peptide substrates: A new diagnostic approach in oncology. Clin Chem 56: 272-280, 2010.

17. Findeisen P, Costina V, Yepes D, Hofheinz R and Neumaier M: Functional protease profiling with reporter peptides in serum specimens of colorectal cancer patients: Demonstration of its routine diagnostic applicability. J Exp Clin Cancer Res 31: 56, 2012.

18. De Pauw B, Walsh TJ, Donnelly JP, Stevens DA, Edwards JE, Calandra T, Pappas PG, Maertens J, Lortholary O, Kauffman CA, et al; European Organization for Research and Treatment of Cancer/Invasive Fungal Infections Cooperative Group; National Institute of Allergy and Infectious Diseases Mycoses Study Group (EORTC/MSG) Consensus Group: Revised definitions of invasive fungal disease from the European Organization for Research and Treatment of Cancer/Invasive Fungal Infections Cooperative Group and the National Institute of Allergy and Infectious Diseases Mycoses Study Group (EORTC/MSG) Consensus Group. Clin Infect Dis 46: 1813-1821, 2008. 
19. Ostrosky-Zeichner L: Invasive mycoses: Diagnostic challenges. Am J Med 125 (Suppl): S14-S24, 2012.

20. Schaal R, Kupfahl C, Buchheidt D, Neumaier M and Findeisen P: Systematic identification of substrates for profiling of secreted proteases from Aspergillus species. J Microbiol Methods 71: 93-100, 2007.

21. Neustadt M, Costina V, Kupfahl C, Buchheidt D, Eckerskorn C, Neumaier M and Findeisen P: Characterization and identification of proteases secreted by Aspergillus fumigatus using free flow electrophoresis and MS. Electrophoresis 30: 2142-2150, 2009.

22. Watson DS, Feng X, Askew DS, Jambunathan K, Kodukula K and Galande AK: Substrate specifity profiling of the Aspergillus fumigatus proteolytic secretome reveals consensus motifs with predominance of Ile/Leu and Phe/Tyr. PLoS One 6: e21001, 2011.

23. Rawlings ND, Morton FR, Kok CY, Kong $\mathrm{J}$ and Barrett AJ: MEROPS: The peptidase database. Nucleic Acids Res 36 (Database): D320-D325, 2008.

24. Findeisen P, Costina V, Yepes D, Hofheinz R and Neumaier M: Functional protease profiling with reporter peptides in serum specimens of colorectal cancer patients: Demonstration of its routine diagnostic applicability. J Exp Clin Cancer Res 31: 56, 2012.

25. Vaideeswar P, Prasad S, Deshpande JR and Pandit SP: Invasive pulmonary aspergillosis: A study of 39 cases at autopsy. J Postgrad Med 50: 21-26, 2004.

26. Findeisen $P$ and Neumaier M: Functional protease profiling for diagnosis of malignant disease. Proteomics Clin Appl 6: 60-78, 2012 .
27. Witt I: Test systems with synthetic peptide substrates in haemostaseology. Eur J Clin Chem Clin Biochem 29: 355-374, 1991.

28. Powell MF, Grey H, Gaeta F, Sette A and Colón S: Peptide stability in drug development: A comparison of peptide reactivity in different biological media. J Pharm Sci 81: 731-735, 1992.

29. Walker JR, Altman RK, Warren JW and Altman E: Using protein-based motifs to stabilize peptides. J Pept Res 62: 214-226, 2003

30. Donnelly JP: Consensus definitions for invasive fungal disease: Strengths, limitations, and revisions. Med Mycol 44 (s1): 285-288, 2006.

31. Subirà M, Martino R, Franquet T, Puzo C, Altés A, Sureda A, Brunet $\mathrm{S}$ and Sierra J: Invasive pulmonary aspergillosis in patients with hematologic malignancies: Survival and prognostic factors. Haematologica 87: 528-534, 2002.

32. Subirà M, Martino R, Rovira M, Vazquez L, Serrano D and De La Cámara R: Clinical applicability of the new EORTC/MSG classification for invasive pulmonary aspergillosis in patients with hematological malignancies and autopsy-confirmed invasive aspergillosis. Ann Hematol 82: 80-82, 2003.

33. Tsitsikas DA, Morin A, Araf S, Murtagh B, Johnson G, Vinnicombe S, Ellis S, Suaris T, Wilks M, Doffman S, et al: Impact of the revised (2008) EORTC/MSG definitions for invasive fungal disease on the rates of diagnosis of invasive aspergillosis. Med Mycol 50: 538-542, 2012.

34. Johnson G, Ferrini A, Dolan SK, Nolan T, Agrawal S, Doyle S and Bustin SA: Biomarkers for invasive aspergillosis: The challenges continue. Biomarkers Med 8: 429-451, 2014. 